\title{
Para-aminobenzoic acid synthase from mushroom Agaricus bisporus enhances UV-C tolerance in Arabidopsis by reducing oxidative DNA damage
}

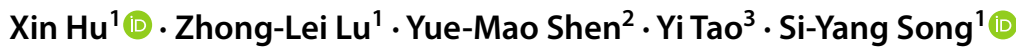

Received: 20 October 2017 / Revised: 17 July 2019 / Accepted: 17 July 2019 / Published online: 9 August 2019

(c) The Author(s) 2019

\begin{abstract}
UV exposure is harmful to plants. Increasing resistance against UV light is thus of great importance to their growth. ParaAminobenzoic acid (PABA) has major roles in many biological processes, involving nucleotide biosynthesis, DNA repair, and DNA methylation, which contributed to UV irradiation. However, no study reports the effect of PABA on UV tolerance, or details of the underlying molecular mechanisms are explored. Hence, the objective of the research is to study the protective effect of PABA on UV in Arabidopsis and explored the molecular mechanisms. We overexpressed PABA synthase gene (Pabs) from Mushroom Agaricus bisporus in Arabidopsis and observed reduced root growth and UV-C hyposensitivity exposed to $2500 \mathrm{~J} \mathrm{~m}^{-2} \mathrm{UV}-\mathrm{C}$ light. UV-C-induced DNA damage was significantly reduced and the expression of decreased DNA methylation 1 (DDM1) was remarkably higher in the Pabs lines, suggesting that overexpression of Pabs may protect against UV-induced DNA damage. In addition, overexpression of Pabs leads to an elevated reactive oxygen species production at root tips and enhanced catalase and superoxide dismutase activity, which may correlate with the enhanced UV tolerance of the Pabs overexpression lines. In summary, overexpression of Pabs from A. bisporus enhances UV-C tolerance of Arabidopsis, suggesting that Pabs takes an important part in defence against DNA damage.
\end{abstract}

Keywords Para-aminobenzoic acid synthase $\cdot$ UV-C tolerance $\cdot$ DNA damage $\cdot$ Reactive oxygen species

Communicated by P. Wojtaszek.

Electronic supplementary material The online version of this article (https://doi.org/10.1007/s11738-019-2936-4) contains supplementary material, which is available to authorized users.

Yi Tao

yitao@xmu.edu.cn

Si-Yang Song

sysong@xmu.edu.cn

1 State Key Laboratory of Cellular Stress Biology, School of Life Sciences, Xiamen University, Fujian, China

2 Key Laboratory of Chemical Biology (Ministry of Education), School of Pharmaceutical Sciences, Shandong University, Shandong, China

3 Xiamen Plant Genetics Key Laboratory, School of Life Sciences, Xiamen University, Fujian, China

\section{Introduction}

Light is crucial to plant growth and development. However, elevated ultraviolet irradiation (UV, 100-290 nm) has detrimental or mutagenic effects on plants, which sometimes can even be lethal (Bray and West 2005; Suchar and Robberecht 2015). UV light can damage plant proteins, lipids, DNA and affect plant development, including inhibition of photosynthesis, increases in ROS, peroxidation of lipids, alteration of metabolism and gene expression (Abdel et al. 2013; Caverzan et al. 2016; Gill et al. 2015; Robson et al. 2015; Suchar and Robberecht 2016). These can result in changes in leaf size, root growth, and apoptotic-like effects (Lee and Suh 2015; Migicovsky and Kovalchuk 2014; Verdaguer et al. 2017). Thus, it is essential to provide insight into the process and mechanism of plant response to UV stress.

To counter the adverse affects of UV radiation on plants, plants have evolved varied protective mechanisms or repair mechanisms. They may reduce the penetration of UV light by means of physiological changes such as alternations in epicuticular waxes (Lee and Suh 2015), production of 
cuticular hairs (Liakopoulos et al. 2006), and formation of leaf glazing (Bruhn et al. 2014). Another strategy to protect themselves from UV damage is the inducible synthesis of UV-absorbing compounds (mainly includes phenolic compounds, flavonoids, and hydroxycinnamate esters) which can act as sunscreen (Lee and Suh 2015). Plants lacking these sunscreens would exhibit enhanced UV sensitivity (Braun et al. 2016).

PABA (para-aminobenzoic acid) is one of the three major precursors for the synthesis of folate, which is an essential element for almost all living organisms. In addition to other functions, folate plays important roles in nucleic acid metabolism and takes part in the processes of DNA methylation and DNA repair (Bhargava and Tyagi 2013). Vasilieva et al. considered PABA as an effective inhibitor of nitric oxideinduced DNA repair pathway in the bacterial cell (Vasilieva et al. 2016). Maintenance of the DNA integrity is crucial to plant in response to DNA damage induced by environment stressors, including UV, ozone, chemical mutagens, and ionizing radiations. In addition, the PABA analog of salicylic acid (SA) has been found to protect pepper (Mahdavian et al. 2008) and soybean (Li et al. 2014) against UV stress. Since the chemical structure of PABA is similar to SA (Sironi et al. 2015), PABA might also take a part in regulating the ultraviolet response of plants. The effects of PABA on UV irradiation damage are still largely unknown. Thus, the first objective of this research is to explore the effect of PABA on UV tolerance in Arabidopsis.

In general, the main mechanism of action of UV on plants is to induce DNA damage. On one hand, UV radiation could directly permeate cell membrane and impair nuclear, chloroplast, and mitochondrial DNA by producing pyrimidine hydrates, pyrimidine dimer and DNA cross-links. On the other hand, UV also indirectly mediated DNA damage in plants by generating ROS and freely reactive radicals. Free radicals like $\mathrm{OH}$ are extremely unstable and susceptible to form oxidative chain reactions, some of which are harmful to the cell. Therefore, our study will also demonstrate the role of PABA in DNA damage induced by UV, including changes in related genes and ROS levels.

In this study, we sub-cloned the open-reading frame (ORF) of Mushroom Agaricus bisporus PABA synthase gene (Pabs) which was previously reported to take a part in heat stress in mushroom (Lu et al. 2014). We overexpressed it in Arabidopsis thaliana and used this genetic tool to investigate the effect of Pabs on environmental stresses in Arabidopsis. We did not observe enhanced heat tolerance in the Pabs overexpressing lines. We also detected the differential expressed genes-related UV resistance, including Arabidopsis thaliana radiation sensitive gene 51 (RAD51), decrease in DNA methylation gene 1 (DDMI), poly-ADP ribose polymerase gene 2 (PARP2) and breast cancer susceptbility gene 1 (BRCA1). In addition, we examined the influence of Pabs on antioxidant capacity after UV-C irradiation. By analyzing the experimental results obtained, we have a conclusion that Pabs plays a role in the protection against damage of Arabidopsis DNA and implicates a new alternative gene for genetic improvement of Arabidopsis UV-C tolerance.

\section{Materials and methods}

\section{Plant material and growth conditions}

The Arabidopsis thaliana ecotype Columbia (Col-0) was obtained from the Arabidopsis Biological Resource Center. Arabidopsis seeds were surface sterilized [75\% $(v / v)$ ethanol] for $5 \mathrm{~min}$ and washed three times in sterile distilled $\mathrm{H}_{2} \mathrm{O}$. Then, seeds were suspended in sterile $0.15 \%(w / v)$ agar to be transferred on Murashige and Skoog (MS) medium plates $[0.4 \%(w / v)$ MS basal medium, 3\% (w/v) sucrose, $0.8 \%(w / v)$ bacteriological agar with $\mathrm{pH}$ 5.8. The plates were sealed and kept at $4{ }^{\circ} \mathrm{C}$ for 2 days for stratification, then flatly put into growth chambers with a $16 / 8 \mathrm{~h}\left(22 / 18^{\circ} \mathrm{C}\right)$ day/night regime.

\section{Generation of Pabs overexpression lines}

The ORF of A. bisporus PABA synthase gene (Pabs, GenBank FJ617437.1) was PCR amplified from the cDNA of mushroom A. bisporus strain 02 by primer: AttB1-pabs, AAAAAGCAGGCTTCATGG CCACCGTTCAACCTCA; AttB2-pabs, AGAAAGCTGGGTTTTAAGAAGATGCAC CAAATC, and then subsequently cloned into the binary vector pEarlygate201 (stored by State Key Laboratory of Cellular Stress Biology, School of Life Science, Xiamen University, Xiamen, P. R. China). The construct was then transformed into Wild-type Col-0 by standard Agrobacterium-mediated techniques. Briefly, $0.1 \mu \mathrm{g}$ plasmid was mixed with thawed electro competent Agrobacterium cells $(50 \mu \mathrm{L})$ in a pre-chilled $2 \mathrm{~mm}$ gap electroporation cuvette ( $25 \mu \mathrm{F}, 2.47 \mathrm{kV}, 400 \Omega, 9 \mathrm{~ms}$ ). The mixture was placed on ice for $5 \mathrm{~min}$ and then added with $1 \mathrm{~mL}$ of fresh LB medium, and recover at $28{ }^{\circ} \mathrm{C}$ for $1 \mathrm{~h}$. Finally, the cells were plated on LB plates (containing $50 \mu \mathrm{g} \mathrm{mL}^{-1}$ of formycin, $10 \mu \mathrm{g} \mathrm{mL}^{-1}$ of gentamycin) and incubated at $28{ }^{\circ} \mathrm{C}$ for 2 days. Positive colonies were screened for PCR identification.

A. thaliana plants were transformed by the floral dip method as described by Clough and Bent (1998). The T1 seeds were harvested. The seedlings were sprayed with $30 \mathrm{mg} \mathrm{L}^{-1}$ glufosinate and screened once a week for a total of three times. The resistant plants continued to be planted to self-pollinated T3 generations. Transgenic T3 lines were identified by homozygote, PCR, and anti-HA western blot analysis. 


\section{Total PABA extraction and HPLC quantitation}

Total PABA extraction was performed as previously described (Lu et al. 2014) with some modification. In brief, $0.2 \mathrm{mg}$ Arabidopsis seedlings were ground fully in liquid nitrogen and extracted three times with $1 \mathrm{~mL}$ of methanol. The three parts of the supernatant were collected together and evaporated. Subsequently, sample was dissolved in $0.5 \mathrm{~mL}$ of $0.1 \mathrm{M}$ sodium acetate buffer ( $\mathrm{pH}$ 5.5), and digested with $0.025 \mathrm{U}$ $\mathrm{mL}^{-1}$ glycosidase (Sigma) to change the conjugated PABA into free type at $30{ }^{\circ} \mathrm{C}$ for $12 \mathrm{~h}$. After that the products were evaporated again. PABA was re-dissolved in $0.5 \mathrm{~mL}$ of $0.1 \mathrm{M}$ sodium citrate ( $\mathrm{pH} 3.7)$ and partitioned against $1.5 \mathrm{~mL}$ of ethyl acetate. PABA could be recovered from the organic phase by back extraction with about $0.5 \mathrm{~mL} 0.05 \mathrm{~N} \mathrm{NaOH}$ solution. After final evaporation, the sample was dissolved in methanol solvent for HPLC quantitation.

\section{UV sensitivity assays}

\section{Shoot assay}

Five-day-old plants were exposed to an appropriate $2500 \mathrm{~J} \mathrm{~m}^{-2}$ UV-C light in UV-cross linker (America UVP Ultraviolet Crosslinkers CL-1000, Shortwave $254 \mathrm{~nm}, 8 \mathrm{~W}, 5$ ultraviolet tubes) and then incubated under dark conditions for 3 days. Plants were then transferred to normal growth conditions and the phenotypes were observed 1 week after UV-C treatment. Sensitivity was assessed by tissue death and leaf yellowing.

\section{Primary root length assay}

Seeds were grown on vertically oriented plates under the same growth conditions mentioned above. After placement in darkness at $4{ }^{\circ} \mathrm{C}$ for 2 days, seedlings were grown vertically at $22{ }^{\circ} \mathrm{C}$ under a $16 / 8 \mathrm{~h}$ light/dark photoperiod. 5-day-old seedlings were divided into control group (without irradiation) and UV group, which was treated with the sub-lethal dose of $1000 \mathrm{~J} \mathrm{~m}^{-2} \mathrm{UV}-\mathrm{C}$ radiation and then incubated under dark conditions for additional 3 days. The seedlings were scanned and root lengths were then measured on scanned images using Scion Image (https://www.scioncorp.com).

\section{DNA damage analysis (comet assay)}

Five-day-old Wild-type Col-0 and Pabs overexpression seedlings were both divided into control group (without irradiation) and UV group, which was treated with the sub-lethal dose of $1000 \mathrm{~J} \mathrm{~m}^{-2} \mathrm{UV}-\mathrm{C}$ radiation. The control group and half of the irradiated plants immediately after exposure were transferred for comet assay procedure or frozen in liquid nitrogen and stored at $-80{ }^{\circ} \mathrm{C}$. The rest of the irradiated plants intended for repair competence studies were transferred to growth chambers under normal growth conditions for additional 7 days to allow plants to repair induced DNA damage. Sample slides were purchased from Trevigen Comet Assay Kit. DNA damage was detected by the neutral comet assay as described previously (Menke et al. 2001). In brief, about $100 \mathrm{mg}$ of the plant material was harvested, briefly rinsed in phosphate buffer saline (PBS, pH 7.0) containing 50 mM EDTA, carefully dried with a paper towel and then immediately used for the comet assay or frozen in liquid nitrogen and stored at $-80{ }^{\circ} \mathrm{C}$. The seedlings were sliced on ice with a fresh razor blade quickly in $300 \mu \mathrm{L}$ PBS with $50 \mathrm{mM}$ EDTA solution and tissue debris removed by filtration through $50 \mu \mathrm{m}$ mesh funnels. $50 \mu \mathrm{L}$ of the resulting suspension was mixed with $150 \mu \mathrm{L}$ of liquid $0.7 \%$ low-melting point agarose (incubated in a $37^{\circ} \mathrm{C}$ water bath). Two drops of the mixture were added quickly on each slide and cooled down on ice. The treated slides were lysed in high salt solution $(2.5 \mathrm{M} \mathrm{NaCl}, 10 \mathrm{mM}$ Tris- $\mathrm{HCl}, \mathrm{pH}$ 9, $100 \mathrm{mM}$ EDTA, $1 \% \mathrm{~N}$-lauroylsarcosinate, $\mathrm{pH}$ 7.6) for $1 \mathrm{~h}$ at $4{ }^{\circ} \mathrm{C}$. After washing for $3 \times 5 \mathrm{~min}$ in electrophoresis buffer (90 mM Tris-borate, $2 \mathrm{mM}$ EDTA, $\mathrm{pH} 8.4$ ) at $4{ }^{\circ} \mathrm{C}$, the electrophoresis was conducted at $30 \mathrm{~V}, 17 \mathrm{~mA}$ for $10 \mathrm{~min}$ at $4{ }^{\circ} \mathrm{C}$. The slides were neutralized with $400 \mathrm{mM}$ Tris- $\mathrm{HCl}(\mathrm{pH} 7.5)$ for $3 \times 5 \mathrm{~min}$, and stained with $50 \mu \mathrm{L}$ SYBR $(1: 10,000)$ for $10 \mathrm{~min}$ at room temperature. Then slides were washed continuously with $70 \%$ ethanol for $2 \times 5 \mathrm{~min}$ and $90 \%$ ethanol for $5 \mathrm{~min}$. Let the slides air drying before the photograph. Images of comets from coded slides were captured at a 20 -fold magnification by epifluorescence. The fraction of DNA in comet tails (\% tail-DNA) was analyzed by comet assay software project (CASP), which serves as a measurement of DNA damage. We examined at least 30 comets in each gel and ran six gel replicas for each sample.

\section{Semi-quantitative and real-time qPCR analysis}

Total RNA was extracted from about $100 \mathrm{mg}$ tissues using TRIzol reagent (Invitrogen) according to the manufacturer's protocol. RNA was DNaseI digested and reverse transcribed as a template for PCR amplification. SYBR Green I (Invitrogen) was added as a fluorescent reporter and LA-Taq Polymerase (Invitrogen) was used. Genespecific primers (Table S1) were used to perform the realtime PCR and semi-quantification PCR in Light Cycler real-time PCR system (ROCHE). Gene expressions were 
normalized to the A. thaliana serine/threonine protein phosphatase 2A.

\section{Western blotting analysis}

By grinding samples in liquid nitrogen, total protein from 7-day-old seedlings was extracted and dissolved in 50 $\mu \mathrm{L}$ protein loading buffer. After $12 \%(w / v)$ SDS-PAGE separation, the proteins were transferred onto PVDF membrane and incubated with the anti-HA (Santa Cruz, USA), respectively, with a dilution of 1:1000 $(v / v)$. Quantity One 1.61 software (Bio-Rad, USA) was used to measure band intensities.

\section{Antioxidant capacity assays}

\section{Reactive oxygen species detection}

The ROS level of the Pabs root cells was measured as previously described (Zhang et al. 2001). Briefly, the Arabidopsis seedlings were incubated for $30 \mathrm{~min}$ in $50 \mu \mathrm{M}$ fluorophore 2, 7-dichlorofluorescin diacetate (H2DCFDA) solutions. After that the roots were rinsed with the phosphate buffer and imaged in the laser scan confocal microscopy (LSCM) using a $488 \mathrm{~nm}$ excitation laser and a $530 \mathrm{~nm}$ emission filter. Optical sections were collected under identical exposure conditions (in manual setup) for all samples. The experiments were repeated at least three times, about 15 samples per treatment.

\section{Antioxidant enzymes activities measurement}

Frozen samples were ground quickly using a pre-chilled mortar and extracted with two volumes of an ice-cold extraction buffer $(50 \mathrm{mM}$ potassium phosphate buffer, $\mathrm{pH} 7.0)$ containing $0.1 \%(w / v)$ ascorbic acid, $1 \mathrm{mM}$ $\mathrm{Na}_{2}$-EDTA, $1 \%(w / v)$ polyvinylpolypirrolidone and $0.1 \%$ $(v / v)$ Triton X-100. The homogenate was then centrifuged at $15,000 \times g$ for $30 \mathrm{~min}$ at $4{ }^{\circ} \mathrm{C}$ and the supernatant was collected to measure protein concentrations using BCA protein assay kit with bovine serum albumin as the standard protein. The enzyme assays were performed by a spectrophotometer. CAT activity was measured with the protocol as described previously (Wang et al. 2015). APX assay was calculated by the method described by Asada (1988). The activity of GR was determined by measuring the oxidation of NADPH at $340 \mathrm{~nm}$ as described by Lee and Lee (2000). The activity of SOD was measured by the method as proposed by Wang et al. (2015).

\section{Statistical analysis}

All experiments were repeated at least three times. Results are shown as means \pm standard error. Data were compared by Student's $t$ test. A $P$ value less than 0.05 was considered to be statistically significant.

\section{Results}

Molecular analysis of Pabs overexpression lines in $A$. thaliana

Three independent $P a b s$ overexpression lines were analyzed (a5, c3 and f2) and found that all lines exhibit high levels in Pabs mRNA levels (Fig. 1a). The increasing levels of PabsHA protein in three transgenic lines were also observed. The Pabs protein content of a5 was higher than that of $\mathrm{c} 3$ (account for $41 \%$ of a5) and f2 (account for $48 \%$ of a5) (Fig. 1b). There was no morphogenical differences between Col-0 and Pabs-overexpressing seedlings (a5) (Fig. 1c, d).

\section{UV enhanced PABA synthesis both in Co1 and Pabs overexpression line}

Our results showed that UV-C exposure led to a rapid induction of PABA contents in both Co1 and Pabs overexpressing Arabidopsis as the irradiation time increased (Fig. 2a). The increase of PABA contents in Pabs overexpressing plants and wild types were 1.6- and 2.4-fold more than those in the control plants at $48 \mathrm{~h}$ after UV irradiation. The background level of PABA in Pabs overexpressing Arabidopsis plants was found to be about two times the level in wild type plants.

\section{Overexpression of Pabs enhances UV tolerance}

To determine the potential for overexpressing Pabs gene to influence UV-C responses, the effect of UV-induced phenotypic changes were compared between $\mathrm{Co} 1$ and Pabs overexpressing Arabidopsis plants. It was found that Col-0 wild-type plants displayed severely bleached cotyledons and retarded growth (Fig. 2b). While transgenic lines a5 with the highest Pabs content exhibited less sensitive to UV radiation, with less suppressed phenotype of growth.

Our results on root growth assay showed that Col-0 and Pabs transgenic seedlings exhibited growth inhibition of the 

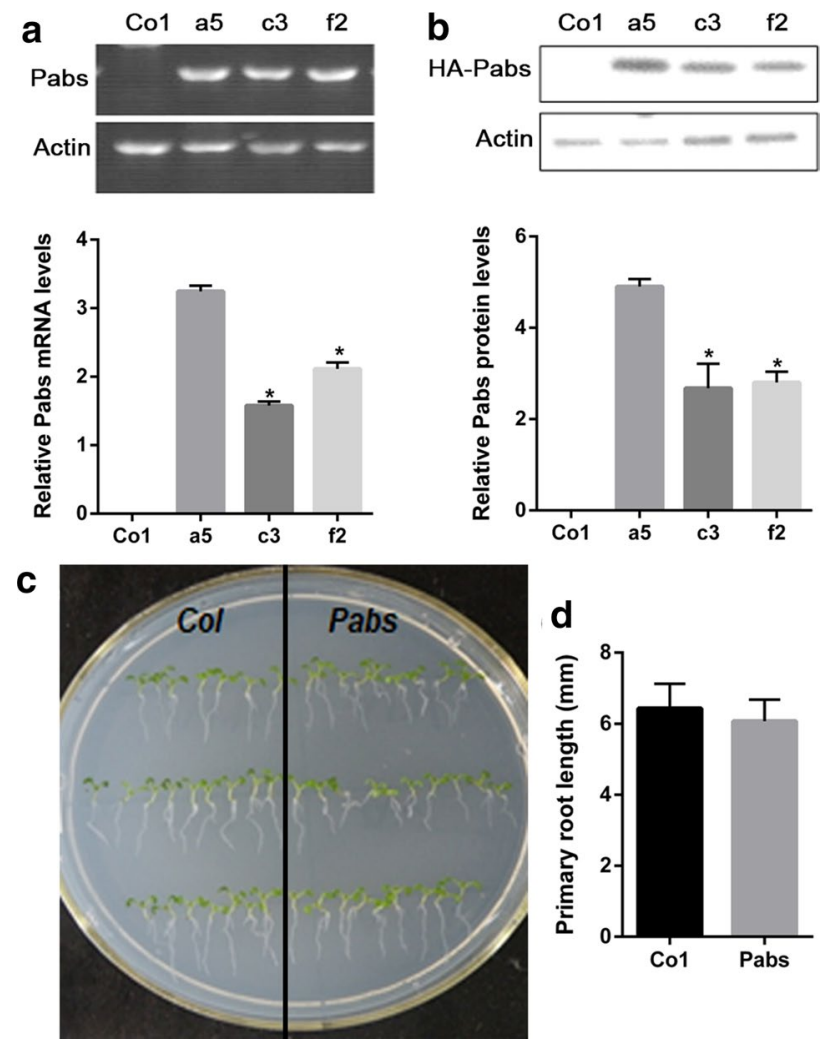

Fig. 1 Characterization of mRNA and protein levels in Co1 and Pabs overexpression lines. a Quantification of total Pabs mRNA level. Data is normalized to the actin loading control $\pm \operatorname{SE}(n=16)$. b Quantification of Pabs-HA protein abundance in overexpression lines. Data are expressed as relative mean band intensity of a five seedling sample normalized to the protein loading control. Morphogenical (c) and primary root length (d) difference between Col-0 and Pabs-overexpressing seedlings. Error bars indicate \pm SE $(n=16)$. *Indicates $P<$ 0.05 relative to a5

primary roots in 3 days after UV irradiation (Fig. 2c). The difference between the irradiated seedlings with the respective unirradiated control becomes greater in both Col-0 and Pabs transgenic lines on the third day. Figure $2 \mathrm{~d}$ shows that the relative root growth (UV-irradiated group/unirradiated control) of Col-0 was significantly lower than that of Pabs transgenic seedlings in 3 days after UV treatment. The relative root growth of Co1 was specifically $20 \%$ less than that of Pabs transgenic lines on the third day. This result showed that Pabs transgenic seedlings exhibited less amount of susceptibility to UV-C during root growth. Thus, Pabs transgenic seedlings were found to be well tolerated under UV-C stress in both shoot and root assays.

\section{Overexpressing Pabs protects plants from UV-C-induced DNA damage}

By evaluating UV-induced DNA damage in Arabidopsis plants, differences were observed between Col-0 and Pabs transgenic seedlings. The result is shown in Fig. 3a. Right after UV-C irradiation, the fraction of DNA migrating in comet tails (tail-DNA) was approximately $80 \%$ and $37 \%$ in wild-type and Pabs overexpression lines, respectively (Fig. 3b). This result indicated that more DNA damage occurs in wild-type plants than in the Pabs overexpression lines. One week after UV-C treatment, the fraction of tailDNA was reduced to $30 \%$ in wild type, while no tail DNA was observed in the Pabs overexpression lines (Fig. 3b). It indicated that plants could repair DNA damage induced by UV-C. However, the results from comet assay actually showed the effects of DNA breaks combined with DNA repair and it reflects the time-effect relationship from DNA damage. Thus, the DNA repair ability was difficult to distinguish between Pabs overexpression lines and Col-0. In summary, our results indicated that overexpressing Pabs can protect plants from UV-C-induced DNA damage.

Supplementary UV-C radiation led to a change in transcripts encoding four DNA damage-related genes, the RAD51, BRCA1, PARP2 and DDM1 (Fig. 3c-f). No significant differences in transcript levels of RAD51, BRCAl and $P A R P 2$ between Col-0 and Pabs overexpression line were observed under normal conditions, but DDM1 mRNA levels in overexpression line were higher than that in wild-type (Fig. 3f). After UV-C irradiation, the relative expression levels of these genes were dramatically altered, but less significant in Pabs overexpression lines. This suggests that less serious DNA damage occurs in Pabs overexpression lines, which was in accordance with previous comet assay data.

\section{Pabs-overexpressing lines represent high levels of ROS and antioxidant enzymes activities}

Results of ROS detection (Fig. 4a) showed that there were higher levels of ROS in the Pabs transgenic roots than in the wild-type roots under normal growth conditions. DCF fluorescence can be detected in the lateral root cap in Pabs overexpression lines, but not in Col-0. UV-C treatment significantly increased ROS level in wild type and the DCF fluorescence altered with local maxima in the lateral root cap. On the other hand, UV-C irradiation induced a minimal increase of ROS production and did not change the pattern of ROS distribution in the Pabs roots. ROS level gradually decreased towards the base of the root and little fluorescence was detected within the elongation zone. 
a

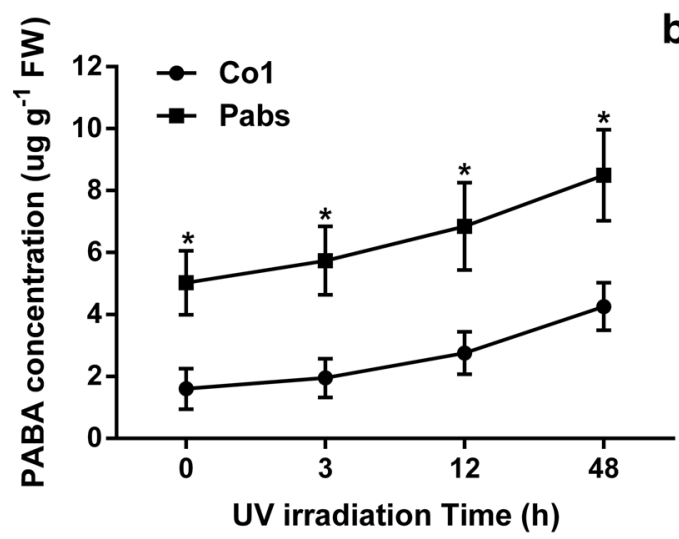

C

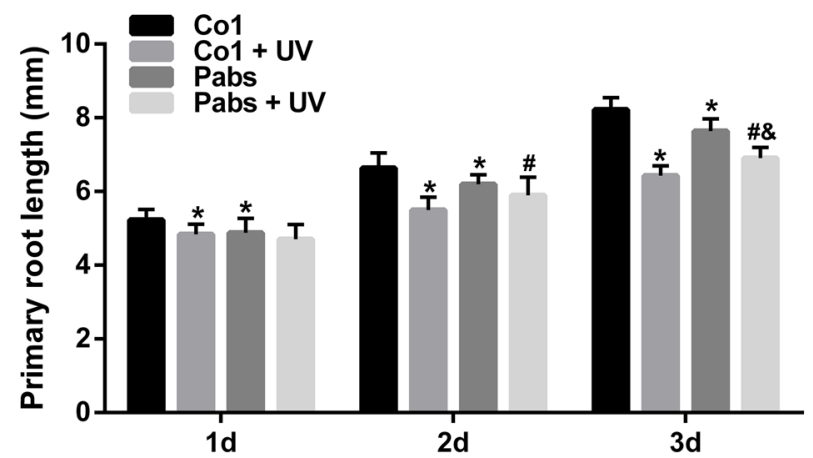

b
Fig. 2 UV tolerance assays. a The total PABA content in wild-type (Co1) and Pabs overexpression lines (a5) seedlings. 12-day-old Arabidopsis seedlings are exposed to UV-C $1000 \mathrm{~J} \mathrm{~m}^{-2}$ and collected at the next four time point $(0,3 \mathrm{~h}, 12 \mathrm{~h}, 48 \mathrm{~h})$. b Overall physiological effects were observed in Arabidopsis lines after $2500 \mathrm{~J} \mathrm{~m}^{-2} \mathrm{UV}-\mathrm{C}$ irradiation. c Primary root length of $\mathrm{Co} 1$ and a5 after exposure to $1000 \mathrm{~J} \mathrm{~m}^{-2} \mathrm{UV}-\mathrm{C}$ irradiation. The average primary roots of 5-dayold Arabidopsis seedlings were valued. d Relative root length of

Activities of the antioxidant enzymes CAT, APX, GR and SOD were shown in Fig. 4b-e. Little changes of CAT activity occurred both in Pabs expression line and Col-0 seedlings $3 \mathrm{~h}$ after UV-C treatment. UV caused a 13\% and 24\% enhancement of SOD activity in Pabs expression line and Col, respectively. Under UV irradiation, APX and GR activity increased 30\% and 59\% in Pabs expression line, with respect to Co1 (27\% and 37\%). These data suggest that after UV-C irradiation, overexpression of Pabs results in more significant improvement in antioxidants activity, which may help Pabs plants efficiently scavenge UV-C-induced ROS.

\section{Discussion}

The roles of $P a b s$ in UV resistance were investigated in $P a b s$ transgenic Arabidopsis plants. In our study, Pabs overexpressing Arabidopsis was less susceptible to UV-C than Col proved by relative primary root elongation assay and the morphogenic responses. Pabs gene was previously proved

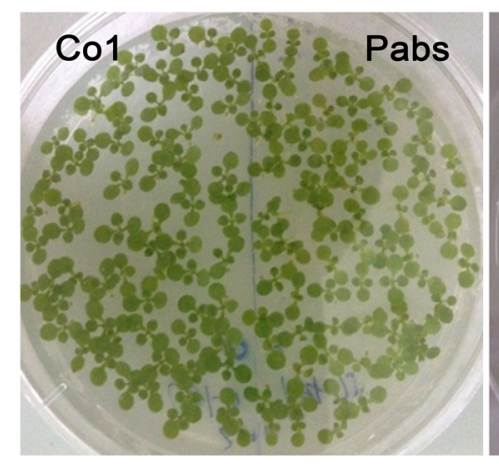

d

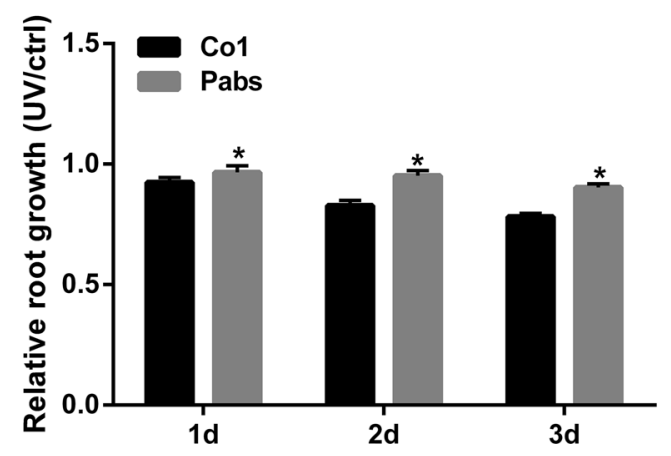

\section{1 week after UV stress}

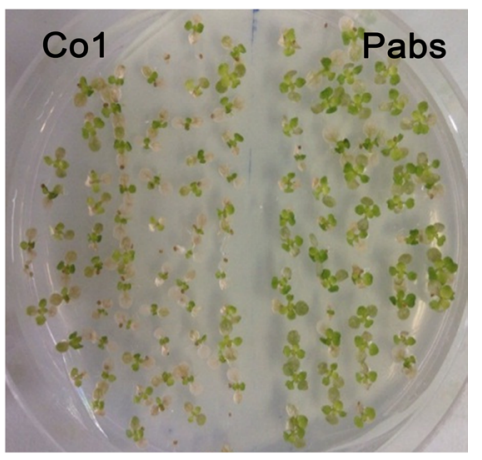

\section{.}

Co1 and a5 exposed to $1000 \mathrm{~J} \mathrm{~m}^{-2}$ UV-C irradiation. Measurements were taken 1, 2 and 3 days after irradiation. Results are expressed as mean of at least three independent experiments. Bars represent standard error $(n=9)$. *Indicates $P<0.05$ relative to Co1 at same time point. "Indicates $P<0.05$ relative to $\mathrm{Co} 1+\mathrm{UV}$ at same time point. \#Indicates $P<0.05$ relative to $P a b s$ at same time point. Results are expressed as mean of three replicates. Bars represent standard error

to be the key enzyme of PABA synthesis in vitro (Deng et al. 2015). While low concentrations of $0.02 \mathrm{mM}$ PABA could slightly increase root length, high concentrations of PABA (more than $0.1 \mathrm{mM}$ ) could significantly inhibit the primary root growth of Arabidopsis seedlings (Crisan et al. 2014). In the present study, we successfully constructed several transgenic Arabidopsis lines for overexpression of Pabs. Comparing to Co1, these lines displayed no obvious morphogenical differences but demonstrated a certain increase in PABA content. It seemed that the growth effect of PABA might not be pronounced by the matters of concentrations. Those lead us to estimate endogenous PABA is mainly via UV-induced signal-transduction pathways to indirectly affect plants growth. Another evidence was that UV caused accumulation of PABA in both Co1 and Pabs overexpression lines, with similar but not significant trends.

Results from UV tolerance study showed that the Pabs overexpression line was resistance. The stability and integrity of the Arabidopsis DNA contents which are highly sensitive to UV exposure have close connection 
a
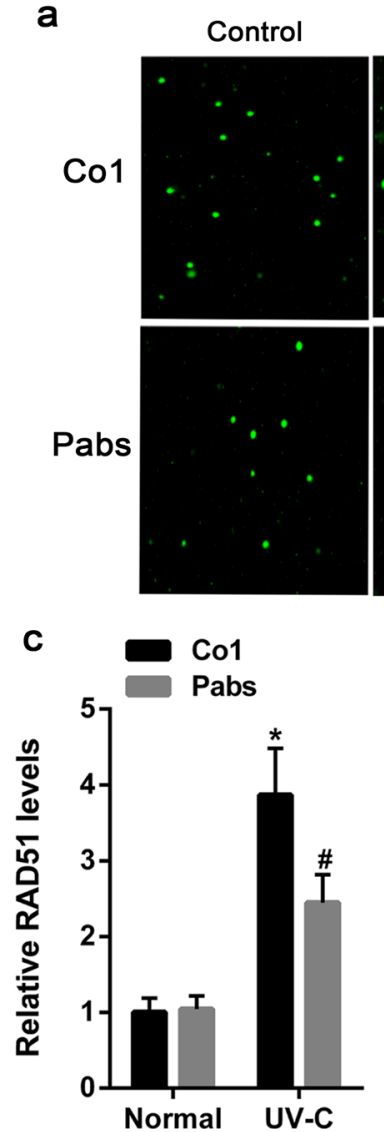

Dirctly after UV 1 week after UV
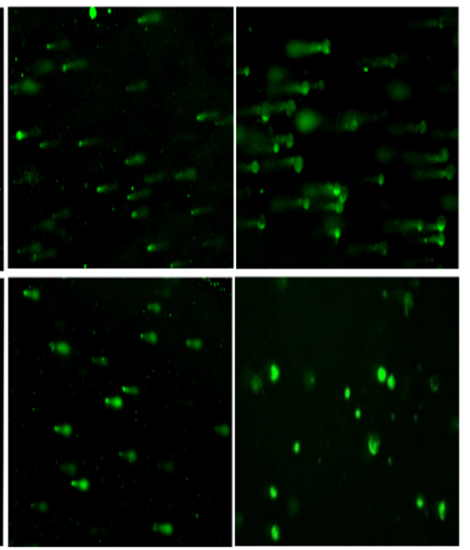

d

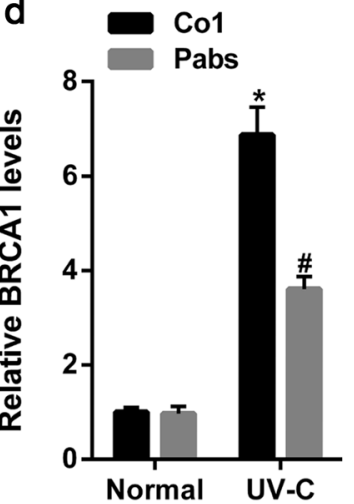

e

Fig. 3 Overexpression of Pabs protects plants from UV-C induced DNA damage. a 7-day-old Pabs overexpressing lines showed less DNA damage compared to Col-0 in response of $1000 \mathrm{~J} \mathrm{~m}^{-2} \mathrm{UV}$ irradiation. The extent of DNA damage is manifested by the fraction of DNA remaining in the comet tails (\% tail DNA) and quantified with the comet assay software project (CASP). b 180 comets of each sample were analyzed by CASP. The select images represented the similar shapes. Pabs comets revealed much less \% tail-DNA content

with UV-resistant morphophysiological traits discovered in Pabs overexpression lines. The aforementioned phenotypic differences between Co1 and Pabs overexpression Arabidopsis indicated that overexpression of exogenous Pabs gene could defend plant DNA against UV stress. These effects of DNA protection and restoration could reduce UV-induced DNA damage, which was important for plant survival under UV irradiation. Besides PABA as a sunscreen absorbs light in the UV-C region, it is also believed that PABA may afford other protection in response to UV irradiation. It was reported that PABA exerts an antioxidant effect after hypoxia by decreasing the abnormally high level of lipid peroxides and stabilizing catalase activity in rat retina (Akberova et al. 1998). Rd Mouse exhibited less oxidative damage in PABA-treated retinas (Galbinur et al. 2009). It was found that PABA reacted rapidly with singlet molecular oxygen $\left({ }^{1} \mathrm{O}_{2}\right)$ and
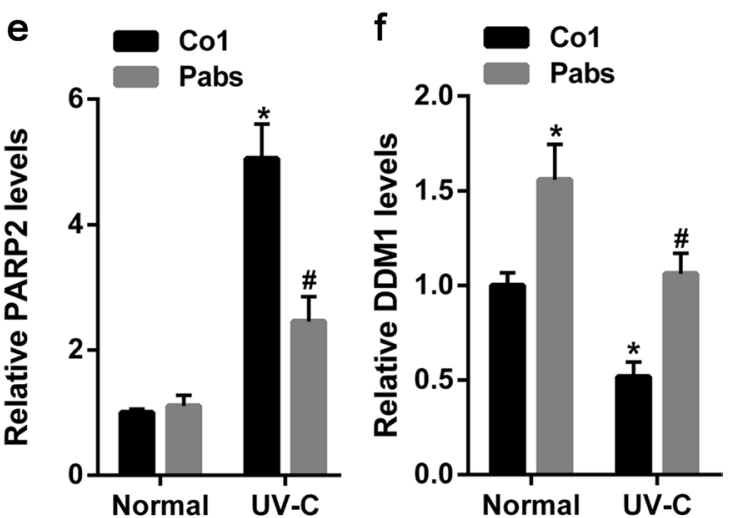

b

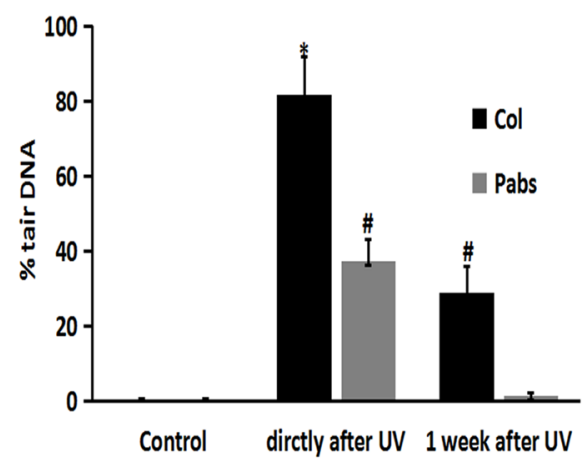

than Col-0. c RAD51, d BRCA1, e PARP2 and f DDM1 mRNA levels changed $3 \mathrm{~h}$ after UV-C exposure in 7-day-old wild-type and overexpression line a5 seedlings. The transcript levels were normalized against PP2A (At1g13320). Values obtained from three independent experiments are reported with the sample SEM. Data show mean values \pm SEM of at least three independent experiments. Values with different letter annotation are significantly different by ANOVA Tukey test with $P<0.05$

HOCI but not $\mathrm{H}_{2} \mathrm{O}_{2}$ and superoxide anions and protected DNA against UV-C-induced damage to calf thymus DNA in vitro (Hu et al. 1995). It was found that PABA could reinforce the repair capacity of thermally induced DNA damage in E. coli $\mathrm{M} 17$ cells, whereas PABA did not prevent DNA damage from heating treatment (Ivanov and Kulikov 1982). Analysis of the molecular and biological mechanisms showed that PABA formed noncovalent complexes with DNA in vitro (Papaneophytou et al. 2014) and activated the major repair enzyme DNA polymerase in $E$. Coli (Vasil'Eva et al. 1982). It indicated that PABA might have an impact on DNA repair.

To minimize the serious consequences of DNA damage, plants adopt complex signaling pathways including arresting cell cycle, activating DNA repair-related genes and synthesizing cellular protective compounds. The evidence of an involvement for Pabs in UV-C damage response 

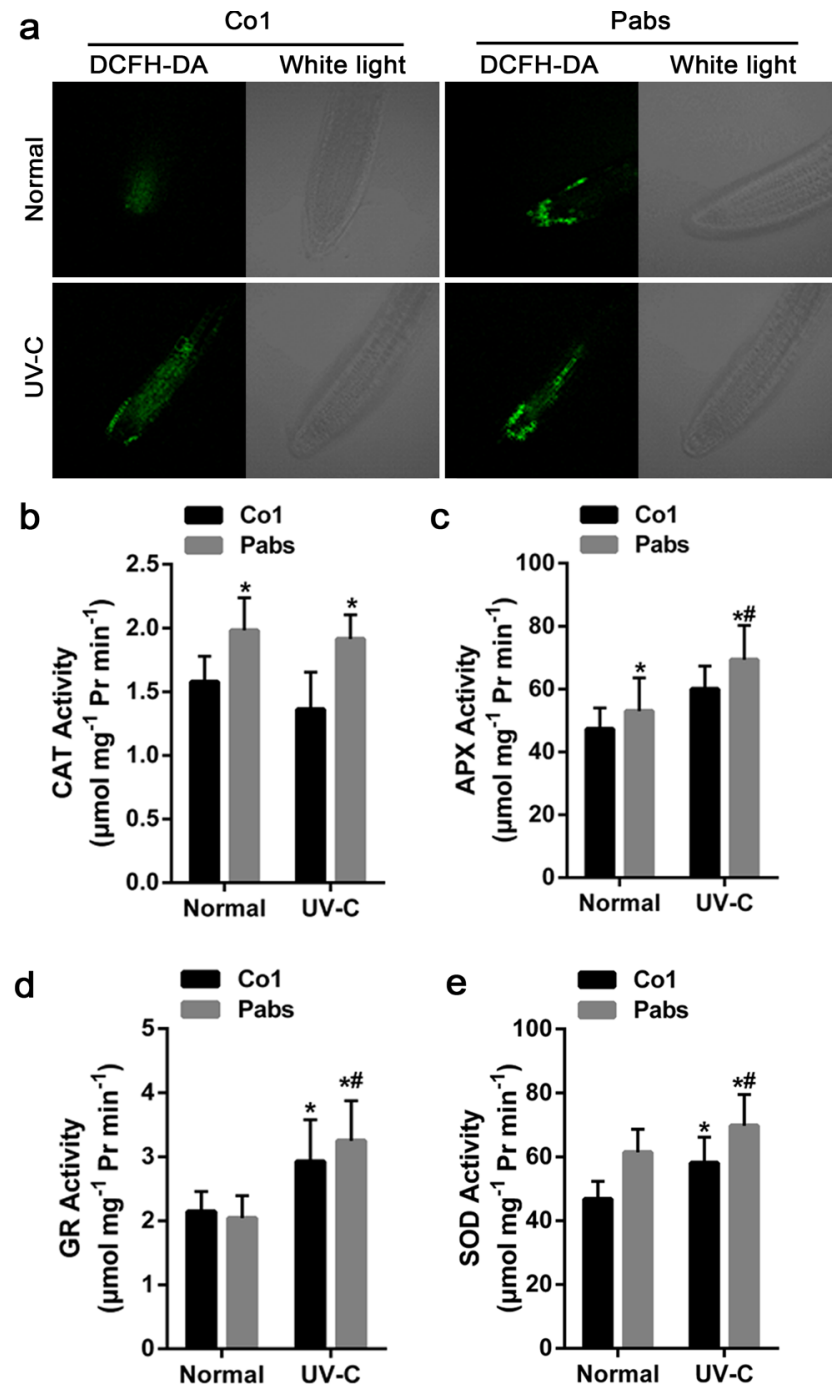

Fig. 4 Pabs-overexpressing lines displayed high levels of ROS and antioxidant enzymes activity. a Overexpression of Pabs increased the ROS level in A. thaliana seedling roots. 7-day-old Arabidopsis seedlings directly (normal) or after exposed to $1000 \mathrm{~J} \mathrm{~m}^{-2} \mathrm{UV}-\mathrm{C}$ (UVC) were imaged using laser scanning confocal imaging. Antioxidant enzymes activity in Col-0 and Pabs plants: CAT (b), APX (c), GR (d) and SOD (e). Arabidopsis seedlings were collected directly (con) or $3 \mathrm{~h}$ after irradiated with $1000 \mathrm{~J} \mathrm{~m}^{-2} \mathrm{UV}-\mathrm{C}$ (UV $3 \mathrm{~h}$ ) for enzyme assays. Results are expressed as mean of three replicates. Bars represent standard error

leads us to explore its role in expression regulation of DNA repair-related genes, including $R A D 51, B R C A 1$, $P A R P 2$ and $D D M 1$ (Fig. 3c-f). These genes are widely regarded as sensors linked to DNA damage response, which could be immediately induced when plant exposure to $\gamma$ and UV-C radiation. Before UV irradiation, there is no difference of RAD51, BRCA1, PARP2 mRNA levels between Co1 and Pabs overexpression line. But after UV irradiation, their mRNA levels all increased, and Pabs transgenic plants exhibited remarkably lower expression levels of RAD51, BRCA1 and PARP2 than Col-0. Interesting for $D D M 1$, which was reduced in the oxidative damage response upon $\gamma$ and UV-C radiation in Arabidopsis, here we found it highly expressed in Pabs overexpression lines in the absence of UV-C, suggesting that $D D M 1$ gene was constitutively induced in these lines (Fig. 3f). DDMI acts as a chromatin-remodeling ATPase participated in maintenance of DNA methylation, histone methylation, gene silencing and homologous recombination (Matzke and Mosher 2014; Shaked et al. 2006; Xie and Shippen 2018). Mutations in DDM1 result in the sensitivity to UV-C radiation (Questa et al. 2013). The increase in DDM1 expression was possibly involved in DNA structure stability and further studies are needed.

Plants DNA could be damaged by direct UV radiation or indirectly oxidative impairment via ROS and free radicals (Blaškovičová et al. 2018). Since PABA functions a potential antioxidant, it is necessary to analyse the ROS generation in Pabs transgenic plants. Under normal conditions, metabolism of ROS is a dynamic equilibrium process between ROS production and scavenging. UV-induced excessive ROS needs to be eliminated by antioxidative enzymes. The high eliminative ability of ROS in plants would effectively reduce oxidative damage and enhance their tolerance to adverse environmental conditions including UV (Wang et al. 2007). Our results demonstrated that Pabs transgenic Arabidopsis possessed elevated levels of ROS. Acclimating to this environment, it also revealed an increase of CAT and SOD activity. Our results on antioxidant enzymes activities measurement showed that APX, GR and SOD except CAT were enhanced after UV-C treatment. Notably, UV-C induced better activity of APX in Pabs overexpression lines. When Pabs plants exposed to UV-C, higher activity of SOD and APX enzymes might quickly take remedial measures to prevent from UVinduced ROS. APX is regarded as efficient scavengers of $\mathrm{H}_{2} \mathrm{O}_{2}$ under stressful conditions because its isoform has much greater affinity of $\mathrm{H}_{2} \mathrm{O}_{2}$ as CAT has (Wang et al. 1999). It was reported that the activity of APX would be increased after UV irradiation in Piceaasperata seedlings (Han et al. 2009). In addition, it was found that $t A P X$ gene overexpression could lead increased tolerance to oxidative damage in both tobacco and Arabidopsis (Caverzan et al. 2012). This study indicated elevated ROS level in Pabs plants contributed to high ability of antioxidation helping them resist UV-C stress.

In conclusion, this study demonstrates that Pabs transgenic Arabidopsis revealed higher tolerance to UV-C stress, compared with the wild-type. Overexpression of Pabs mediated ROS generation and induced antioxidase activity. Overexpressing Pabs protects plants from UV$\mathrm{C}$-induced DNA damage which could be the reason of improved tolerance of Pabs plants. Further studies are 
needed to provide genetic evidence of the details in PABAinduced ROS generation and to search the critical signaling components between PABA function and UV-C stress.

Author contributions statement Conceived and designed the experiments: XH, ZL, Y-MS, YT and S-YS. Performed the experiments: XH, ZL. Analyzed the data: XH, ZL. Wrote the paper: $\mathrm{XH}, \mathrm{YT}$.

\begin{abstract}
Acknowledgements We are grateful to Professor Yue-Mao Shen for constructive discussions about the manuscript, to Professor Xian-ming Deng, Professor Zhong-huiZheng, Professor Lian-ru Zhang, Professor Yaojian Huang, and Vice Professor Qing-yanXu for valuable suggestions, and also to Senior experimentalist Zhi-yu Hu for technical support with the experiment. We would also like to thank Dr. Zhong-Lei $\mathrm{Lu}$ and Dr. Li-Xin Deng for their generous help and encouragement. This study was supported by the National Nature Science Foundation of China (NSFC No. 30571259).
\end{abstract}

Open Access This article is distributed under the terms of the Creative Commons Attribution 4.0 International License (http://creativeco mmons.org/licenses/by/4.0/), which permits unrestricted use, distribution, and reproduction in any medium, provided you give appropriate credit to the original author(s) and the source, provide a link to the Creative Commons license, and indicate if changes were made.

\section{References}

Abdel HE, Abdullah H (2013) Alhuqail AA (2013) Oxidative damage and mutagenic potency of fast neutron and UV-B radiation in pollen mother cells and seed yield of Vicia faba L. Biomed Res Int 9:824656

Akberova SI, Musaev Galbinur PI, Magomedov NM, Babaev KF, Gakhramanov KM, Stroeva OG (1998) Comparative assessment of antioxidant activity of para-aminobenzoic acid and emoxipin in retina. Vestn Oftalmol 114(6):39

Asada K (1988) Production, scavenging and action of active oxygen. Tanpakushitsu Kakusan Koso 33(16):2659-2664

Bhargava S, Tyagi SC (2013) Nutriepigenetic regulation by folatehomocysteine-methionine axis: a review. Mol Cell Biochem 387(1-2):55-61

Blaškovičová J, Sochr J, Koutsogiannis A, Diamantidou D, Kopel P, Adam V, Labuda J (2018) Detection of ROS generated by UV-C irradiation of CdS quantum dots and their effect on damage to chromosomal and plasmid DNA. Electroanalysis 30(4):698-704

Braun C, Reef R, Siebeck UE (2016) Ultraviolet absorbing compounds provide a rapid response mechanism for UV protection in some reef fish. J Photochem Photobiol B Biol 160:400-407

Bray CM, West CE (2005) DNA repair mechanisms in plants: crucial sensors and effectors for the maintenance of genome integrity. New Phytol 168(3):511-528

Bruhn D, Mikkelsen TN, Rolsted MM, Egsgaard H, Ambus P (2014) Leaf surface wax is a source of plant methane formation under UV radiation and in the presence of oxygen. Plant Biol 16(2):512-516

Caverzan A, Passaia G, Rosa SB, Ribeiro CW, Lazzarotto F, Margispinheiro M (2012) Plant responses to stresses: role of ascorbate peroxidase in the antioxidant protection. Genet Mol Biol 35(4 suppl):1011-1019

Caverzan A, Casassola A, Brammer SP (2016) Antioxidant responses of wheat plants under stress. Genet Mol Biol 39(1):1-6
Clough SJ, Bent AF (1998) Floral dip: a simplified method for Agrobacterium-mediated transformation of Arabidopsis thaliana. Plant J 16(6):735-743

Crisan ME, Bourosh P, Maffei ME, Forni A, Pieraccini S, Sironi M, Chumakov YM (2014) Synthesis, crystal structure and biological activity of 2-hydroxyethylammonium salt of $p$-aminobenzoic acid. PLoS One 9(7):e101892

Deng L, Shen Y, Song S (2015) Cloning, expression, and characterization of para-aminobenzoic acid (PABA) synthase from Agaricus bisporus 02, a thermotolerant mushroom strain. J Microbiol Biotechnol 25(1):66-73

Galbinur T, Obolensky A, Berenshtein E, Vinokur V, Chowers I, Chevion M, Banin E (2009) Effect of para-aminobenzoic acid on the course of retinal degeneration in the rd10 mouse. J Ocul Pharmacol Ther 25(6):475-482

Gill SS, Anjum NA, Ritu G, Manoranjan J, Narendra T (2015) DNA damage and repair in plants under ultraviolet and ionizing radiations. Sci World J 2015:250158

Han C, Liu Q, Yang Y (2009) Short-term effects of experimental warming and enhanced ultraviolet-B radiation on photosynthesis and antioxidant defense of Picea asperata seedlings. Plant Growth Regulat 58(2):153-162

Hu ML, Chen YK, Chen LC, Sano M (1995) Para-aminobenzoic acid scavenges reactive oxygen species and protects DNA against UV and free radical damage. J Nutr Biochem 6(6):504-508

Ivanov SD, Kulikov SV (1982) Effect of $p$-aminobenzoic acid and aminobenzhydrazide on DNA repair. Bull Exp Biol Med 4(93):427-429

Lee DH, Lee CB (2000) Chilling stress-induced changes of antioxidant enzymes in the leaves of cucumber: in gel enzyme activity assays. Plant Sci 159(1):75-85

Lee SB, Suh MC (2015) Advances in the understanding of cuticular waxes in Arabidopsis thaliana and crop species. Plant Cell Rep 34(4):557-572

Li X, Ma L, Bu N, Li Y, Zhang L (2014) Effects of salicylic acid pretreatment on cadmium and/or UV-B stress in soybean seedlings. Biol Plant 1(58):195-199

Liakopoulos G, Stavrianakou S, Karabourniotis G (2006) Trichome layers versus dehaired lamina of Olea europaea leaves: differences in flavonoid distribution, UV-absorbing capacity, and wax. Environ Exp Bot 3(55):294-304

Lu Z, Kong X, Lu Z, Xiao M, Chen M, Zhu L, Shen Y, Hu X, Song $S$ (2014) Para-aminobenzoic acid (PABA) synthase enhances thermotolerance of mushroom Agaricus bisporus. PLoS One 9(3):e91298

Mahdavian K, Ghorbanli M, Kalantari KM (2008) Role of salicylic acid in regulating ultraviolet radiation-induced oxidative stress in pepper leaves. Rus J Plant Physiol 4(55):560

Matzke MA, Mosher RA (2014) RNA-directed DNA methylation: an epigenetic pathway of increasing complexity. Nat Rev Genet 15(6):394

Menke M, Chen I, Angelis KJ, Schubert I (2001) DNA damage and repair in Arabidopsis thaliana as measured by the comet assay after treatment with different classes of genotoxins. Mutat Res 493(1-2):87-93

Migicovsky Z, Kovalchuk I (2014) Transgenerational changes in plant physiology and in transposon expression in response to UV-C stress in Arabidopsis thaliana. Plant Signal Behav 9(11):e976490

Papaneophytou CP, Grigoroudis AI, Mcinnes C, Kontopidis G (2014) Quantification of the effects of ionic strength, viscosity, and hydrophobicity on protein-ligand binding affinity. Acs Med Chem Lett 5(8):931-936

Questa JI, Fina JP, Casati P (2013) DDM1 and ROS1 have a role in UV-B induced- and oxidative DNA damage in A. thaliana. Front Plant Sci 4:420 
Robson TM, Klem K, Urban O, Jansen MAK (2015) Re-interpreting plant morphological responses to UV-B radiation. Plant Cell Environ 38(5):856-866

Shaked H, Avivi-Ragolsky N, Levy AA (2006) Involvement of the Arabidopsis SWI2/SNF2 chromatin remodeling gene family in DNA damage response and recombination. Genetics 173(2):985-994

Sironi A, Marinotto D, Riccardi C, Zanini S, Guerrini E, Pina CDEA (2015) Effect of salicylic acid and 5-sulfosalicylic acid on uv-vis spectroscopic characteristics, morphology, and contact angles of spin coated polyaniline and poly(4-aminodiphenylaniline) thin films. J Spectrosc 2015:2015

Suchar VA, Robberecht R (2015) Integration and scaling of UV-B radiation effects on plants: from DNA to leaf. Ecol Evolut 5(13):2544-2555

Suchar VA, Robberecht R (2016) Integration and scaling of UV-B radiation effects on plants: from molecular interactions to whole plant responses. Ecol Evolut 6(14):4866

Vasil'Eva SV, Tonkal' TE, Gorodetskii SI, Rapoport IA (1982) Genetic activity of para-aminobenzoic acid: The intensification of DNA polymerase I-dependent repair induced by chemical mutagens in toluene-treated Escherichia coli cells. Genetika 18(3):392-398

Vasilieva SV, Petrishcheva MS, Gusarova EI, Osipov AN (2016) Vitamin para-aminobenzoic acid (PABA) controls generation of nitric oxide (NO) in vitro and its biological functions in the bacterial cells. Adv Tech Biol Med 4:195
Verdaguer D, Jansen MA, Llorens L, Morales LO, Neugart S (2017) UV-A radiation effects on higher plants: exploring the known unknown. Plant Sci 255:72-81

Wang J, Zhang H, Allen RD (1999) Overexpression of an Arabidopsis peroxisomal ascorbate peroxidase gene in tobacco increases protection against oxidative stress. Plant Cell Physiol 40(7):725-732

Wang Y, Wisniewski M, Meilan R, Cui M, Fuchigami L (2007) Transgenic tomato (Lycopersicon esculentum) overexpressing $c A P X$ exhibits enhanced tolerance to UV-B and heat stress. J Appl Hortic $8(2): 4$

Wang J, Zhang H, Zhang T, Zhang R, Liu R, Chen Y (2015) Molecular mechanism on cadmium-induced activity changes of catalase and superoxide dismutase. Int J Biol Macromol 77:59-67

Xie X, Shippen DE (2018) DDM1 guards against telomere truncation in Arabidopsis. Plant Cell Rep 37(3):501-513

Zhang X, Zhang L, Dong F, Gao J, Galbraith DW, Song CP (2001) Hydrogen peroxide is involved in abscisic acid-induced stomatal closure in Vicia faba. Plant Physiol 126(4):1438-1448

Publisher's Note Springer Nature remains neutral with regard to jurisdictional claims in published maps and institutional affiliations. 